'Departamento de Cirugía, Facultad de Medicina, Universidad de Concepción. Concepción, Chile.

${ }^{2}$ Centro Cardiovascular, Hospital Guillermo Grant Benavente de Concepción. Concepción, Chile.

${ }^{3}$ Departamento de Salud

Pública, Facultad de Medicina,

Universidad de Concepción.

Concepción, Chile.

${ }^{4}$ Departamento de Kinesiología. Facultad de Medicina. Universidad de Concepción. Concepción, Chile.

aEstudiante de la carrera de Kinesiología.

Trabajo no recibió finaciamiento. Los autores declaran no tener conflictos de interés.

Recibido el 1 de abril de 2019, aceptado el 26 de junio de 2019 .

Correspondencia a: Dr. Enrique Seguel Soto Departamento de Cirugía,

Facultad de Medicina Universidad de Concepción Avenida Roosevelt esquina Janequeo. Concepción, Chile. enseguel@udec.cl

\section{Resultados a 5 años de la revascularización exclusiva con dos arterias mamarias en pacientes con enfermedad coronaria multivaso}

\author{
ENRIQUE SEGUEL S. ${ }^{1,2}$, ALECK STOCKINS L. ${ }^{1,2}$, \\ ROBERTO GONZÁLEZ L. ${ }^{1,2}$, ALDO VERA-CALZARETTA ${ }^{3}$, \\ ALEXIS GONZÁLEZ-BURBOA ${ }^{3}$, ALEJANDRO HIDALGO B., ${ }^{4, a}$
}

\section{Exclusive coronary revascularization with both mammary arteries. Analysis of 73 patients}

Background: Exclusive coronary revascularization with both mammary arteries could result in lower rates of adverse events in the long term. Aim: To describe the five-year evolution of a cohort of patients operated on with this technique. Material and Methods: Follow up analyzing survival of 73 patients aged $59 \pm 9$ years ( $82 \%$ men) who underwent exclusive coronary surgery with two mammary arteries between December 1, 2010 and April 12, 2017. We studied their clinical characteristics, surgical results, operative morbidity and mortality and adverse events up to June 30, 2018. Results: Six patients had two-vessel lesions and 67 three-vessel lesions. The operative risk calculated by additive and logistic EuroSCORE was 2.5 and 2.3\%, respectively. A mean of 3.75 anastomoses / patient were performed, 116 with left mammary artery ( 73 to the anterior descending artery, 38 to a diagonal artery and 5 for other objectives) and 158 with right mammary artery (69 to a first marginal artery, 23 to a second marginal artery and 64 to posterior descending artery). There was one case of mediastinitis and one (1.5\%) patient died. The mean follow-up was $64.6 \pm 23.7$ months. The 5-year survival was $90.4 \%$. Conclusions: Coronary revascularization with two exclusive mammary arteries allowed a complete revascularization of the heart with a low rate of complications and adverse effects at five years.

(Rev Med Chile 2019; 147: 718-726)

Key words: Cardiovascular Surgical Procedures; Coronary Artery Bypass; Mammary Arteries; Coronary Disease.
L a arteria mamaria interna (AMI) es el injerto de elección para la revascularización de la arteria descendente anterior del corazón, debido a su mayor permeabilidad y asociación de su uso con una mejor sobrevida y menos eventos en el largo plazo que los injertos venosos ${ }^{1-3}$. Teóricamente, la incorporación de un segundo injerto de similares características debería asociarse a mejores resultados. En efecto, varios estudios han mostrado que el uso de dos arterias mamarias se asoció a una mejor sobrevida comparado con el uso de una sola, incluso en pacientes añosos. Este beneficio se observa en el largo plazo, después de los 10 años de seguimiento ${ }^{4-8}$.

A pesar de esa evidencia, el uso de doble arteria mamaria en cirugía coronaria es poco frecuente. 
En Estados Unidos de Norteamérica y Europa corresponde a menos de $5 \%$ del total de cirugías coronarias $^{9-11}$.

Un inconveniente del uso de dos arterias mamarias es que la mamaria derecha pediculada solo puede ser utilizada en ramas proximales de la arteria circunfleja (pasando por el seno transverso) o el tronco de la coronaria derecha.

En 1991, J. A. Barra propuso seccionar la mamaria derecha y anastomosarla a la mamaria izquierda en "Y", permitiendo revascularizar todos los territorios del corazón con el uso de anastomosis secuenciales ${ }^{12,13}$. Este mismo autor mostró buenos resultados clínicos y angiográficos a un año de seguimiento ${ }^{14}$.

En 2007, el equipo de la Clínica Marie Lannelongue, en París, publicó los resultados del control angiográfico precoz de una serie de pacientes operados con la técnica, mostrando una excelente permeabilidad tanto de la "Y" como de las anastomosis distales ${ }^{15}$.

El equipo de cardiocirugía del Hospital Guillermo Grant Benavente inició el uso de esta técnica a fines de 2010, con el objetivo de evaluar sus ventajas en términos de lograr una revascularización arterial completa y una baja tasa de eventos en el seguimiento. Los resultados inmediatos y hasta 39 meses fueron publicados en $2015^{16}$.

El propósito de este estudio es reportar la evolución a 5 años de este grupo, incorporando algunos pacientes intervenidos hasta la realización de este estudio.

\section{Material y Métodos}

Estudio descriptivo de los resultados operatorios, la sobrevida y eventos cardiovasculares mayores a 5 años de los pacientes intervenidos de cirugía coronaria exclusiva con dos arterias mamarias en "Y" (BIMA-Y), entre el $1^{\circ}$ de diciembre de 2010 y el 12 de abril de 2017, en el Hospital Guillermo Grant Benavente $(\mathrm{n}=73)$.

Los datos fueron extraídos del registro de cirugías cardiacas del centro cardiovascular, la base de datos del hospital y los registros del laboratorio de hemodinamia, construyéndose una base de datos manteniendo los casos anónimos.

\section{Selección de pacientes}

La selección de pacientes para la técnica se realizó considerando la anatomía coronaria (lesiones proximales $>75 \%$, vasos coronarios de calibre $>1,5 \mathrm{~mm}$, no calcificados, no intramiocárdicos).

\section{Técnica quirúrgica}

Todas las intervenciones se realizaron con anestesia general por esternotomía media. Ambas arterias mamarias internas se disecaron con técnica esqueletizada y sin abrir las pleuras (Figura 1).

Una vez completada la disección de ambos injertos, se procedió a la heparinización sistémica, a la canulación arterial en la aorta ascendente y venosa atrio - cava. Sin entrar en circulación extracorpórea, se exploró el corazón e identificaron los objetivos a revascularizar.

Se seccionaron la mamaria izquierda a distal y la mamaria derecha a proximal y distal, y se construyó una anastomosis entre ambas de forma término-lateral en "Y" con sutura de polipropileno 8-0 (Prolene ${ }^{\circledR}$, Ethicon, Johnson \& Johnson, Brasil) (Figura 2).

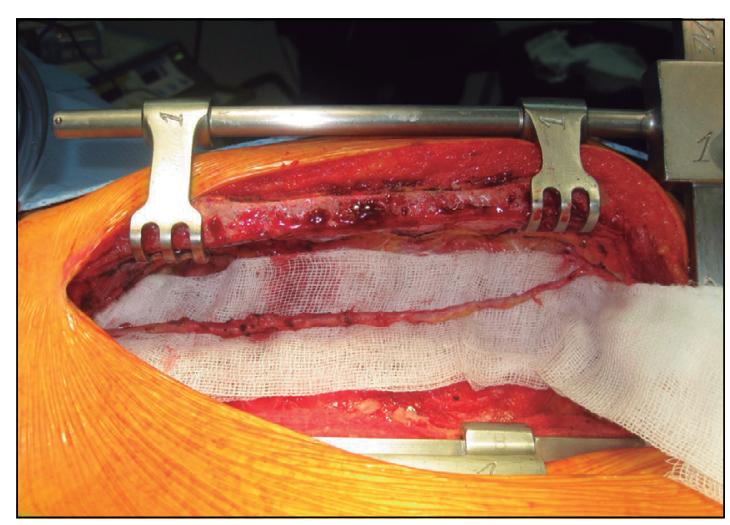

Figura 1. Arteria mamaria interna disecada con técnica esqueletizada.

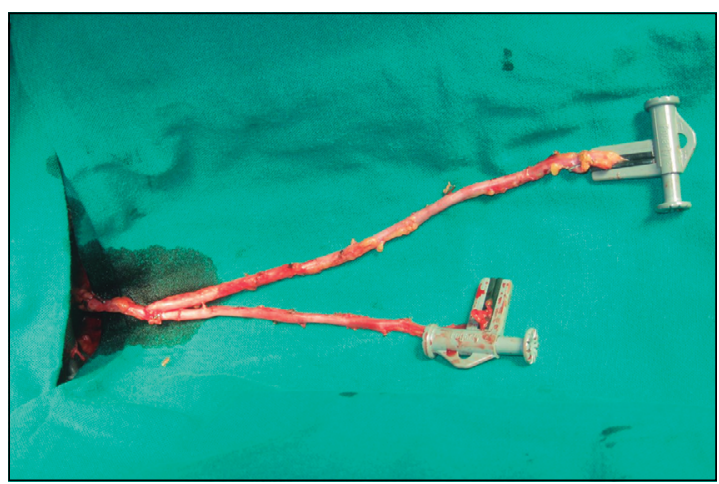

Figura 2. Construcción de la anastomosis en "Y" entre las dos arterias mamarias. 
La revascularización de la cara anterior se realizó utilizando la mamaria izquierda, mediante la confección de anastomosis latero-laterales en las ramas diagonales y término-lateral en la descendente anterior (Figura 3).

La revascularización de la cara lateral se realizó con la mamaria derecha mediante la confección de anastomosis latero-laterales en las ramas marginales, y la cara inferior mediante la confección de anastomosis término-lateral sobre la arteria descendente posterior (Figura 4).

Se consideró revascularización completa a la confección, durante la cirugía, de anastomosis a todos los vasos que fueron planeados antes de la cirugía.

El detalle de la técnica quirúrgica utilizada puede encontrarse en la publicación previa de nuestro grupo ${ }^{17}$.

\section{Variables analizadas}

Se tabularon las características clínicas y demográficas de los pacientes, el riesgo operatorio calculado por EuroSCORE aditivo y logístico ${ }^{18,19}$, la extensión de la enfermedad coronaria, el número y objetivos de las anastomosis distales, duración (en minutos) de la intervención, horas de ventilación mecánica postoperatoria, débito de los drenajes durante las primeras $24 \mathrm{~h}$ y necesidad de transfusión de hemoderivados.

Las complicaciones se definieron como: quirúrgicas (hemorragia, reoperación), neurológicas (accidente vascular encefálico, déficit transitorio,

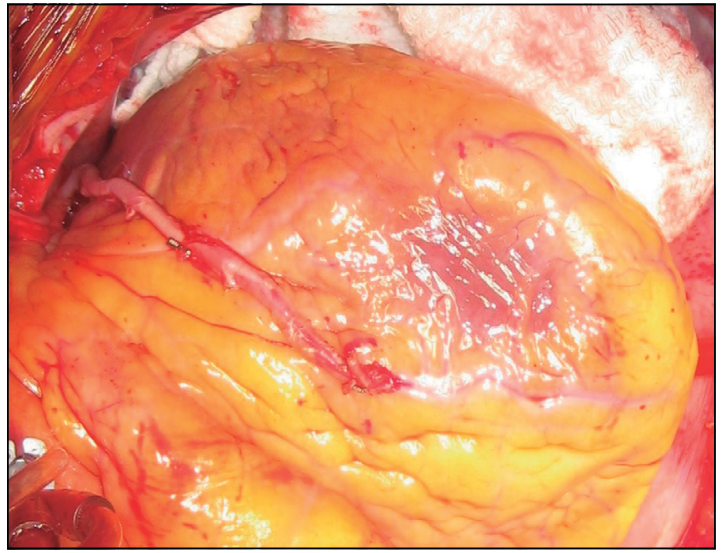

Figura 3. Anastomosis de la arteria mamaria interna izquierda sobre arterias de la pared anterior del corazón. coma), renales (insuficiencia renal aguda con o sin necesidad de diálisis), cardiacas (arritmias, infarto perioperatorio, insuficiencia cardiaca), infecciones (herida operatoria, mediastinitis, extremidad inferior, infección urinaria), pulmonares (ventilación mecánica $>48$ h, neumonía), vasculares (disección aórtica, isquemia extremidad) y otras (digestivas).

La mortalidad operatoria se consideró hasta los 30 días posterior a la cirugía o hasta el alta hospitalaria, si el paciente permaneció más de un mes hospitalizado.

\section{Seguimiento}

El seguimiento se completó hasta el 30 de junio de 2018.

La sobrevida y causas de muerte se obtuvieron de acuerdo a los datos del Servicio de Registro Civil e Identificación.

Se estudiaron, por una parte, la mortalidad alejada, identificando aquella de causa cardiovascular (secundaria a infarto, insuficiencia cardiaca, arritmias o paro cardiorrespiratorio) y no cardiovascular (trauma, neoplasias, infecciones, etc.) y, por otra, los eventos cardiovasculares mayores: infarto agudo al miocardio (IAM), accidente cerebro vascular (AVE) y reintervención.

No se realizó coronariografía de control a nuestros pacientes y solo se estudiaron aquellos que tuvieron indicación por clínica (síntomas, eventos agudos).

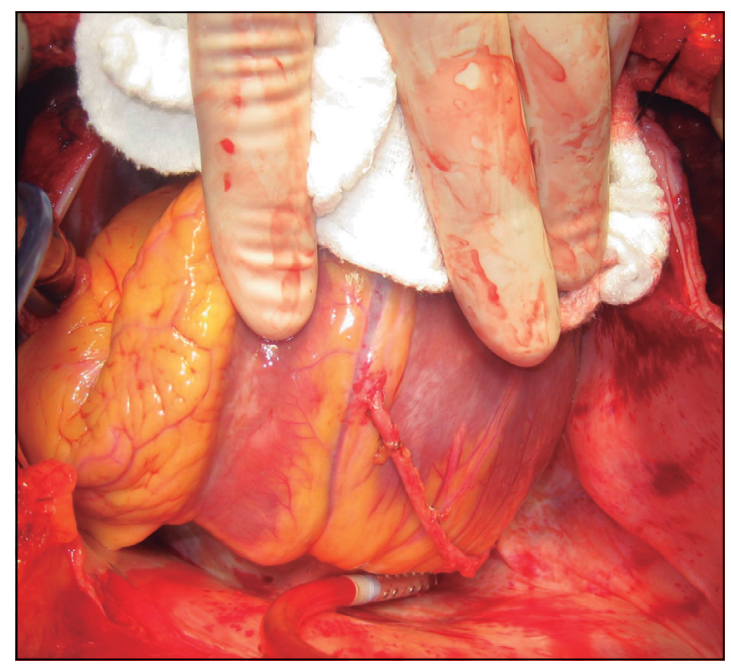

Figura 4. Anastomosis de la arteria mamaria interna derecha con la arteria descedente posterior (pared inferior del corazón). 


\section{Análisis estadísticos}

Se realizó un análisis descriptivo univariado utilizando gráficos y tablas para describir las distribuciones porcentuales de variables nominales $\mathrm{u}$ ordinales. Se utilizaron medidas de tendencia central y de dispersión para las variables cuantitativas. Se utilizó el método Kaplan Meier para el estudio de la función de supervivencia global, de causas cardiacas y otras causas.

Para el procesamiento estadístico de los datos se utilizaron los programas estadísticos IBM SPSS 21.0 y Stata 14.

\section{Aspectos éticos}

La realización de este estudio fue aprobada por el Comité Ético Científico del Servicio de Salud Concepción.

\section{Resultados}

\section{Pacientes}

Durante el período de estudio se realizaron 1.418 cirugías coronarias exclusivas en el centro. Se utilizó doble mamaria en $167(11,8 \%)$ pacientes, y la técnica BIMA-Y en $73(5,1 \%)$.

Las características de los pacientes se muestran en la Tabla 1.

\section{Cirugías}

Se confeccionaron 272 anastomosis distales, con promedio de 3,75 $\pm 0,6$ puentes/paciente. Estas correspondieron a 116 anastomosis con mamaria izquierda (73 a la descendente anterior, 38 a una rama diagonal y 5 a otro objetivo) y a 156 anastomosis con mamaria derecha (69 a una $1^{\circ}$ marginal, 23 a una $2^{\circ}$ marginal y 64 a una interventricular posterior). Se realizó un procedimiento asociado en un paciente (reemplazo valvular aórtico).

Se extubaron en pabellón 63 pacientes (94\%). La duración promedio de la ventilación mecánica en los otros 4 fue 3,5 h (rango 1- $6 \mathrm{~h}$ ).

Los resultados de la cirugía se muestran en la Tabla 2.

\section{Complicaciones}

El débito de los drenajes en las primeras $24 \mathrm{~h}$ fue de $671 \pm 310 \mathrm{ml}$ (rango 210-1.590 ml). El débito fue mayor a $1.000 \mathrm{ml}$ en 11 pacientes. Recibieron transfusión de hemoderivados 11
Tabla 1. Características de los pacientes

\begin{tabular}{|c|c|}
\hline \multicolumn{2}{|l|}{ Sociodemográficas } \\
\hline \multicolumn{2}{|l|}{ Sexo, n (\%) } \\
\hline Masculino & $60(82,2)$ \\
\hline Femenino & $13(17,8)$ \\
\hline Edad, $\overline{\mathbf{x}} \pm \mathrm{DE}$ (años) & $59,0 \pm 8,7$ \\
\hline \multicolumn{2}{|l|}{ Factores de riesgo cardiovascular } \\
\hline Hipertensión arterial, n (\%) & $56(76,7)$ \\
\hline Diabetes mellitus, n (\%) & $30(41,1)$ \\
\hline Tabaquismo, n (\%) & $30(41,1)$ \\
\hline Dislipidemia, n (\%) & $21(28,8)$ \\
\hline Accidente vascular encefálico, n (\%) & $3(4,1)$ \\
\hline Insuficiencia renal crónica, n (\%) & $1(1,4)$ \\
\hline \multicolumn{2}{|l|}{ Historia cardiovascular } \\
\hline Angina inestable, n (\%) & $11(15,1)$ \\
\hline Infarto reciente (< 90 días), n (\%) & $22(30,1)$ \\
\hline Angioplastía previa, n (\%) & $7(9,6)$ \\
\hline \multicolumn{2}{|l|}{ Lesiones coronarias } \\
\hline Lesión de dos vasos, n (\%) & $6(8,2)$ \\
\hline Lesión de tres vasos, n (\%) & $67(91,8)$ \\
\hline Fracción de eyección* ${ }^{\star} \bar{x} \pm D E$ & $58,5 \pm 9,8$ \\
\hline \multicolumn{2}{|l|}{ Riesgo operatorio } \\
\hline EuroSCORE aditivo**,$\overline{\mathrm{x}} \pm \mathrm{DE}$ & $2,5 \pm 2,1$ \\
\hline EuroSCORE logístico***, $\bar{x} \pm \mathrm{DE}$ & $2,3 \pm 2,3$ \\
\hline
\end{tabular}

*Rango: 35-75\%; **Rango: 0-10 puntos; ${ }^{* * * R a n g o: ~ 0,88-~}$ $16,8 \%$.

Tabla 2. Cirugías

$\begin{array}{|lr|}\begin{array}{l}\text { Número de bypass } \mathrm{n}(\%) \\ 3\end{array} & 24(32,9) \\ 4 & 43(58,9) \\ 5 & 6(8,2) \\ \text { Anastomosis distal LIMA, n (\%) } & 32(43,8) \\ 1 & 39(3,4) \\ 2 & 2(2,7) \\ 3 & 1(1,4) \\ \text { Anastomosis distal RIMA, n (\%) } & 59(80,8) \\ 1 & 13(17,8) \\ 2 & 1(1,4) \\ 3 & 175,4 \pm 37,3 \\ \text { Cirugía asociada, n (\%) } & 1,4 \pm 5,3 \\ \text { Duración de la cirugía (min), } \bar{x} \pm \text { DE }\end{array}$


$(15,1 \%)$ pacientes. No hubo reintervenciones por sangrado.

Se presentaron complicaciones médicas en 8 y quirúrgicas en 7 pacientes. No hubo complicaciones neurológicas, renales ni ventilación mecánica prolongada.

Falleció 1 paciente $(1,5 \%)$ debido a una mediastinitis.

El detalle de la morbimortalidad operatoria se presenta en la Tabla 3.

\section{Seguimiento}

Se logró el seguimiento de $100 \%$ de los casos, con un promedio de 64,6 meses (DE: 23,7; rango 14-90).

Fallecieron 9 pacientes (a los 10, 14, 21, 44, 46, $46,62,69$ y 80 meses). La sobrevida global de la serie fue de $90,4 \%$ a 5 años. La curva de sobrevida global se muestra en la Figura 5.

Tres pacientes fallecieron de causas cardiovasculares (paro cardiorrespiratorio en uno e insuficiencia cardiaca descompensada en dos). Los otros 6 pacientes fallecieron de causas no-cardiovasculares: tres por neumonias, uno por un síndrome urémico, uno por un traumatismo encefalocraneano y uno por una neoplasia. La sobrevida a 5 años excluyendo los fallecidos de causas no cardiovasculares fue de 95,6\%. La Figura 6 muestra la curva de sobrevida considerando solo los fallecidos de causa cardiovascular.

\section{Tabla 3. Complicaciones postoperatorias}

\begin{tabular}{|c|c|}
\hline Transfusiones, n (\%) & $11(15,9)$ \\
\hline Drenaje en 24 h, $\bar{x} \pm$ DE (ml) & $671,4 \pm 309,8$ \\
\hline \multicolumn{2}{|l|}{ Complicaciones renales, n (\%) } \\
\hline Falla renal aguda & $2(2,7)$ \\
\hline Hipokalemia & $1(1,4)$ \\
\hline \multicolumn{2}{|l|}{ Complicaciones quirúrgicas, n (\%) } \\
\hline Anemia grave & $1(1,4)$ \\
\hline Dehiscencia de herida operatoria & $1(1,4)$ \\
\hline Dehiscencia de esternotomía & $1(1,4)$ \\
\hline Edema agudo pulmonar & $1(1,4)$ \\
\hline Hipotensión & $2(2,7)$ \\
\hline Reoperación por sangrado & $0 \quad(0)$ \\
\hline \multicolumn{2}{|l|}{ Complicaciones cardiacas, n (\%) } \\
\hline Fibrilación auricular & $4(5,5)$ \\
\hline Hipotensión & $2(2,7)$ \\
\hline Bloqueo AV, marcapaso & $1(1,4)$ \\
\hline Tromboembolismo pulmonar & $1(1,4)$ \\
\hline \multicolumn{2}{|l|}{ Complicaciones infecciosas, n (\%) } \\
\hline Infección de herida operatoria & $5(6,8)$ \\
\hline Mediastinitis & $1(1,4)$ \\
\hline Días UCl, $\bar{x} \pm D E$ & $3,1 \pm 6,1$ \\
\hline Días alta, $\bar{x} \pm \mathrm{DE}$ & $9,6 \pm 7,7$ \\
\hline Mortalidad operatoria, n (\%) & $1(1,4)$ \\
\hline
\end{tabular}

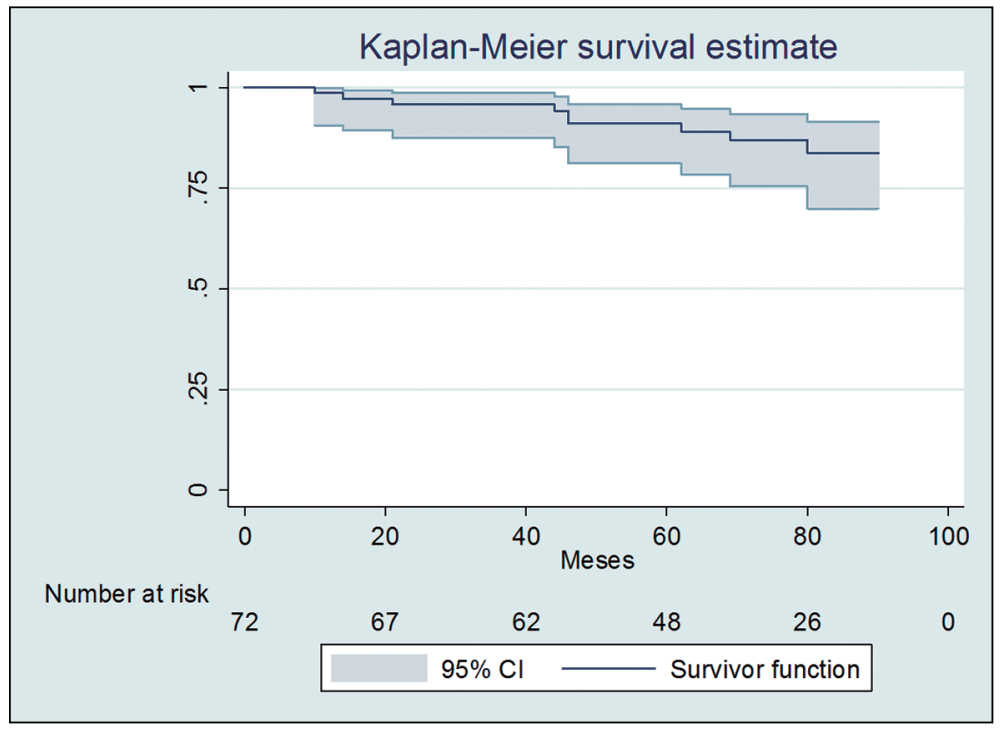

Figura 5. Curva de sobrevida global. 


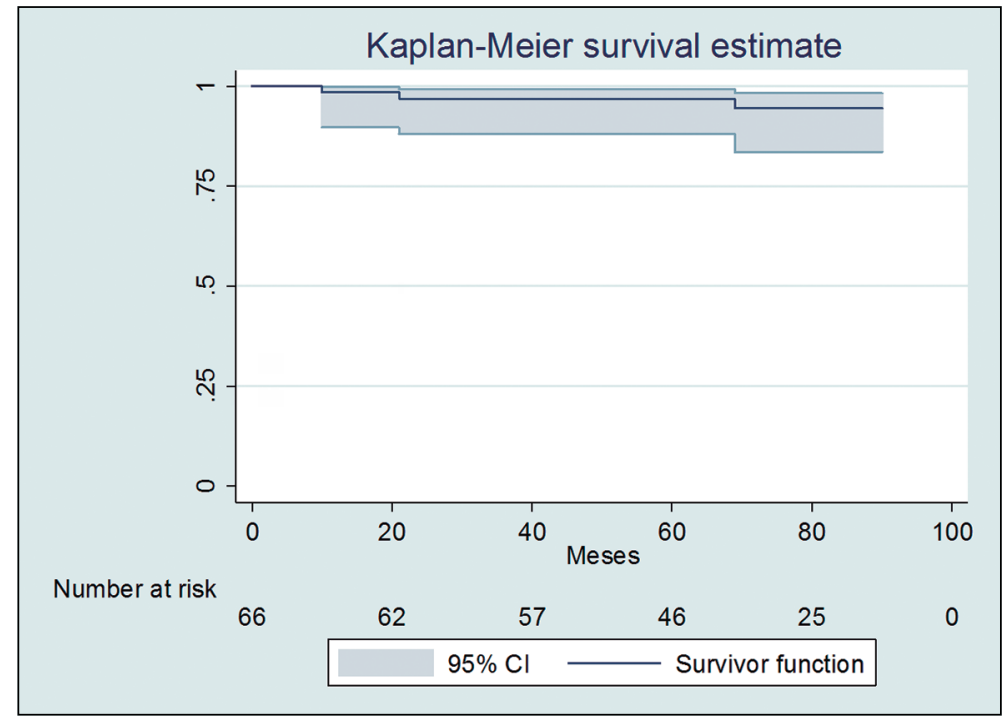

Figura 6. Curva de sobrevida libre de muerte cardiovascular.
Durante el seguimiento, ningún paciente presentó un infarto agudo al miocardio, ni accidente vascular encefálico.

Dos pacientes requirieron de una reintervención por síntomas: uno fue estudiado con coronariografía a los 7 meses y se encontraron todos los puentes permeables. Otro se estudió a los 20 meses, encontrando una oclusión de la AMI izquierda luego de la anastomosis en "Y". Se realizó una angioplastia e implante de stent a la descendente anterior.

\section{Discusión}

En esta serie de cirugías coronarias utilizando la técnica de dos arterias mamarias en "Y" se logró una revascularización de todos los objetivos con una baja morbimortalidad operatoria y eventos en el seguimiento a cinco años.

La técnica propuesta por Barra ${ }^{12}$ permitió aumentar al doble el alcance de la AMI derecha, alcanzar las arterias de la pared lateral e inferior del corazón y lograr la revascularización completa con estos injertos. Debido a que la anastomosis entre ambas mamarias puede comprometer la revascularización de todo el corazón, y a la necesidad de realizar anastomosis secuenciales, esta técnica es más demandante que la cirugía tradicional.
En la selección de pacientes privilegiamos aquellos más jóvenes y de bajo riesgo operatorio. Si bien la serie incluye a 6 pacientes mayores de 70 años, 52\% eran menores de 60 años. El riesgo operatorio calculado por EuroSCORE aditivo mostró un bajo riesgo en $38(56,7 \%)$ casos, riesgo moderado en $24(38,8 \%)$ y riesgo alto en $8(11,9 \%)$. Al observar a los pacientes coronarios operados en el mismo período en nuestro hospital, el promedio de edad fue 62 años, siendo el EuroSCORE aditivo de riesgo bajo en $37,7 \%$, moderado en $41,5 \%$ y alto en $20,8 \%$. Sin embargo, debido a lo pequeño de la serie, no es posible hacer una comparación entre las diferentes técnicas y pacientes.

Se eligieron pacientes con lesiones coronarias mayores a $75 \%$ para evitar el fenómeno de competencia de flujo. Este problema puede afectar a los injertos arteriales, los que tienden a atrofiarse cuando son anastomosados a arterias con lesiones menos significativas, y en los que el flujo por la arteria nativa es mayor que el flujo por el injerto ${ }^{20,21}$.

Se logró la revascularización de todos los objetivos planeados con la técnica, con un promedio de 3,7 puentes por paciente. La duración promedio de la cirugía $(3 \mathrm{~h})$ no se extendió por la necesidad de disecar ambas mamarias. Esta técnica tiene como ventaja, además, una menor necesidad de 
manipulación de la aorta, lo que asociado al uso de pinzamiento único de la aorta, disminuye el riesgo de embolías cerebrales.

A pesar del riesgo de mediastinitis, se intervinieron 30 pacientes diabéticos. La idea fue realizar una revascularización arterial en estos pacientes utilizando la disección equeletizada para disminuir el riesgo de esta complicacion. Durante el postoperatorio se trataron con insulina para mantener glicemias normales. Hubo una mediastinitis en la serie, que corresponde, además, a la paciente que falleció, y cinco infecciones superficiales de la herida operatoria. El riesgo de esta complicación es una de las razones que explican el poco uso de dos arterias mamarias, especialmente en pacientes añosos, obesos y diabéticos. La técnica de disección esqueletizada de la mamaria ha demostrado disminuir el riesgo de estas complicaciones ${ }^{22-25}$. El estudio ART, que comparó en forma aleatorizada a dos grupos de pacientes intervenidos con una y dos arterias mamarias, confirmó una mayor tasa de complicaciones de la esternotomía en los pacientes operados con dos mamarias comparado con aquellos en los cuales se utilizó una sola (1,9\% vs $0,6 \%)$. Sin embargo, estas complicaciones no afectaron ni la mortalidad a 30 días $(1,2 \%$ en ambos grupos) ni a un año (2,5\% vs $2,3 \%$ respectivamente ${ }^{26}$.

La sobrevida global a cinco años fue de $90 \%$, con $95,6 \%$ libre de muerte cardiovascular. Esto es similar a los resultados publicados para la cirugía coronaria estándar, sin observar un beneficio de esta técnica en términos de sobrevida en ese plazo. Los resultados a 5 años del estudio ART no mostraron diferencias en las sobrevidas gobales entre el uso de una y dos arterias mamarias $(91,6 \%$ versus $91,3 \%$, respectivamente, $p=0,77)^{27}$. Los resultados finales de este estudio a 10 años tampoco mostraron diferencias en la mortalidad alejada para los pacientes intervenidos con mamaria única y doble $(21,2 \%$ y $20,4 \%$ respectivamente; HR 0,96 ; IC $95 \% 0,82-1,12 ; \mathrm{p}=0,58)$. El análisis posterior mostró que un número importante de los pacientes aleatorizados a una mamaria recibieron un segundo injerto arterial con arteria radial $(22 \%)$ y que a 10 años existiría un beneficio con el uso de dos injertos arteriales (no necesariamente dos mamarias) ${ }^{28}$.

Pese a que el presente estudio no demostró un beneficio del uso de dos mamarias a 5 años, es importante considerar que el beneficio del esta técnica se observa a largo plazo. El estudio multicéntrico publicado por Irribarra y cols, que incluyó casi 48.000 pacientes intervenidos entre 1992 y 2014, que fueron seguidos por 13 años, mostró una sobrevida a 5 años de $92,4 \%$ para doble mamaria y de $91,7 \%$ para una. La sobrevida a 10 años fue $82,6 \%$ versus $79,8 \%$, y a 15 años fue de $71,6 \%$ y $63,2 \%$, respectivamente, demostrando un beneficio en la sobrevida que solo es observable a largo plazo $(\mathrm{p}=0,001)$. Este estudio observó, además, una menor tasa de revascularización repetida durante el seguimiento (19,4 versus $15,1 \%$, $\mathrm{p}=0,004)^{29}$.

Dos pacientes de la serie fueron reestudiados, encontrando en uno de ellos todos los puentes permeables y una oclusión de la AMI izquierda posterior a la anastomosis con la AMI derecha, lo que probablemente se debe a un problema técnico. El paciente recibió una angioplastia con implante de stent en la arteria descendente anterior. Esto es similar a lo observado en otras series publicadas con esta técnica ${ }^{15,30}$.

Finalmente, es importante destacar que esta es la primera serie nacional en la que se realiza una revascularización completa con dos arterias mamarias, con resultados observados similares a los publicados en otras series internacionales.

\section{Limitaciones}

Se trata de una serie de pacientes de bajo riesgo seleccionados para ser intervenidos con esta técnica en un solo centro. El estudio invasivo de pacientes asintomáticos no está indicado, por lo que no se hizo estudio angiográfico y se desconoce la permeabilidad real de los injertos. Por otro lado, el seguimiento clínico a cinco años es insuficiente para observar los beneficios de la técnica.

\section{Conclusión}

La cirugía coronaria exclusiva con dos arterias mamarias en "Y" permitió la revascularización de todos los territorios del corazón con una baja tasa de complicaciones y de eventos a cinco años. Se debe continuar el seguimiento de esta serie para determinar si se pueden observar las ventajas esperadas para la técnica. 


\section{Referencias}

1. Lytle B, Loop F, Cosgrove D, Ratliff N, Easley K, Taylor P. Long Term (5 To 12 Years) Serial Studies Of Internal Mammary Artery And Saphenous Vein Coronary Bypass Grafts. J Thorac Cardiovasc Surg 1985; 89: 24858.

2. Loop F, Lytle B, Cosgrove D, Stewart R, Goormastic M, Williams G, et al. Influence Of The Internal Mammary Artery Graft On 10 Years Survival And Other Cardiac Events. N Eng J Med 1986; 314: 1-6.

3. Cameron A, Davis C, Green G, Schaff H. Coronary Bypass Surgery With Internal Thoracic Artery Grafts, Effects On Survival Over A 15 Year Period. N Eng J Med 1996; 334: 216-9.

4. Fiore A, Naunheim K, Dean P, Kaiser G, Pennington G, Willman V, et al. Results of Internal Thoracic Artery Grafting Over 15 Years: Single versus Double Grafts. Ann Thorac Surg 1990; 49: 202-9.

5. Lytle B, Blackstone E, Loop F, Houghtaling P, Arnold J, Akhrass R, et al. Two Internal Thoracic Artery Grafts Are Better Than One. J Thorac Cardiovasc Surg 1999; 117: 855-72.

6. Moran S, Irarrazaval MJ, Zalaquett R, Villavicencio M, Garayar B, Muñoz C, y cols. Revascularización miocárdica con arteria mamaria interna bilateral. Rev Chil Cardiol 1996; 15: 4-8.

7. Moran S, Irarrazaval MJ, Zalaquett R, Villavicencio M, Garayar B, Muñoz C, et al. Revascularización miocárdica con una y dos arterias mamarias: resultados clínicos y seguimiento alejado. Rev Med Chile 1997; 125: 391-401.

8. Lytle B, Blackstone E, Sabik J, Houghtaling P, Loop F, Cosgrove D. The Effect of Bilateral Internal Thoracic Artery Grafting on Survival During 20 Postoperative Years. Ann Thorac Surg; 2004; 78: 2005-14.

9. Puskas JD. "Why did you not use both internal thoracic arteries?”. Circulation 2012; 126: 2915-17.

10. Tabata M, Grab JD, Khalpey Z, Edwards FH, O’Brien $\mathrm{SM}$, Cohn LH et al. Prevalence and variability of internal mammary artery graft use in contemporary multivessel coronary artery bypass graft surgery: analysis of the Society of Thoracic Surgeons National Cardiac Database. Circulation 2009; 120: 935-40.

11. El Bardissi AW, Aranki SF, Sheng S, O’Brien SM, Greenberg CC, Gammie JS. Trends in isolated coronary artery bypass grafting: an anlysis of the Society of Thoracic Surgeons adult cardiac surgery database. J Thorac Cardiovasc Surg 2012; 143: 273-81.

12. Barra JA, Mondine P, Bezon E, Mahlab A, Rukbi I, Braesco J. Revascularisation des artéres coronaires. Réimplantation en $\mathrm{Y}$ de l'artére mammaire interne droite darts l'artére mammaire interne gauche. Presse Med 1991; 20: 423-5.

13. Barra JA, Mondine P, Mahlab A, Bezon E, Rukbi I, Slimane AK, et al. Right internal mammary artery reimplantation into the left internal mammary artery: Y anastomosis. 25 cases. Ann Chir 1991; 45: 661-6.

14. Barra JA, Bezon E, Mansourati J, Rukbi I, Mondine P, Youssef Y. Reimplantation of the right internal thoracic artery as a free graft into the left in situ internal thoracic artery (y procedure) One-year angiographic Results. J Thorac Cardiovasc Surg 1995; 109: 1042-8.

15. Azmoun A, Ramadan R, Al-Attar N, Kortas CH, Ghostine $S$, Caussin C, et al. Exclusive Internal Thoracic Artery Grafting in Triple-Vessel-Disease Patients: Angiographic Control. Ann Thorac Surg 2007; 83: 2098-102.

16. Seguel E, Stockins A, Figueroa F, González R, Alarcón E, Quiñonez C. Cirugía Coronaria Exclusiva Con Dos Mamarias En "Y": Resultados A Corto Plazo. Rev Chil Cardiol 2016; 34 (3): 182-9.

17. Seguel E, González R, Stockins A, Alarcón E, Cárdenas P. Técnicas quirúrgicas. Revascularización miocárdica completa con dos mamarias. Rev Chil Cir 2012; 64 (2): 210-4.

18. Nashef SA, Roques F, Michel P, Gauducheau E, Lemeshow S, Salamon R. European System For Cardiac Operative Risk Evaluation (Euroscore). Eur J Cardiothorac Surg 1999; 16: 9-13.

19. Roques F, Michel P, Goldstone AR, Nashef SA. The Logistic Euroscore. Eur Heart J 2003; 24: 882-3.

20. Moran SV, Baeza R, Guarda E, Zalaquett R, Irarrazaval MJ, Marchant E, et al. Predictors of radial artery patency for coronary bypass operations. Ann Thorac Surg 200; 72: $1552-6$.

21. Tatoulis J. Total arterial coronary revascularization-patient selection, stenoses, conduits, targets. Ann Cardiothorac Surg 2013; 2: 499-506.

22. Lytle B. Skeletonized internal thoracic artery grafts and wound complications. J Thorac Cardiovasc Surg 2001; 121: 695-6.

23. He G, Ryan W, Acuff T, Bowman R, Douthit M, Yang $\mathrm{Ch}$, et al. Risk factors for operative mortality and sternal wound infection in bilateral internal mammary artery grafting. J Thorac Cardiovasc Surg 1994; 107: 196-202.

24. Ridderstolpe L, Gill H, Ahlfeldt H, Rutberg H. Superficial and deep sternal wound complications: incidence, risk factors and mortality. Eur J Cardiothorac Surg 2001; 20: 1168-75.

25. Matsa M, Paz Y, Gurevitch J, Shapira I, Kramer A, Pevny D, et al. Bilateral skeletonized internal thoracic artery grafts in patients with diabetes mellitus. J Thorac Cardiovasc Surg 2001; 121: 668-74. 
26. Taggart D, Altman D, Gray A, Lees B, Nugara F, Yu $\mathrm{LM}$, and the ART Investigators Randomized trial to compare bilateral vs single internal mammary coronary artery bypass grafting: 1-year results of the Arterial Revascularisation Trial (ART). Eur Heart J 2010; 31: 2470-81.

27. Taggart D, Altman D, Gray A, Lees B, Gerry S, Benedetto U, Flather M, for the ART Investigators* . Randomized Trial of Bilateral versus Single Internal-Thoracic-Artery Grafts . N Engl J Med 2016; 375: 2540-9.

28. Taggart D, Benedetto U, Gerry S, Altman D, Gray A, Lees B, et al, for the Arterial Revascularization Trial Investigators. Bilateral versus Single Internal-Thora-
cic-Artery Grafts at 10 Years. N Engl J Med 2019; 380: 437-46.

29. Iribarne A, Schmoker J, Malenka D, Leavitt B, McCullough J, Weldner P et al. Does Use of Bilateral Internal Mammary Artery Grafting Reduce Long-Term Risk of Repeat Coronary Revascularization? A Multicenter Analysis. Circulation 2017; 136: 1676-85.

30. Glineur D, Hanet C, Poncelet A, D'hoore W, JC Funken, J Rubay, et al. Comparison of Bilateral Internal Thoracic Artery Revascularization Using In Situ or Y Graft Configurations A Prospective Randomized Clinical, Functional, and Angiographic Midterm Evaluation. Circulation 2008; 118 [suppl 1]: S216-21. 